\title{
Retour d'expérience de la sécheresse 2003
}

\section{H. DE SOLÈRE}

MEDAD, DPPR

20 avenue de Ségur

75302 Paris 07 SP

Actuellement MEDAD,

CERTU VOLSECUR

helene.de-solere@

equipement gouv.ft

Une synthẻse sur la sécheresse de l'èté 2003 est présentée. L'accent est mis sur les questions nouvelles apportées par cet événement qui a conduit à développer de nouvelles approches sur la façon de prendre en compte le risque de sécheresse géotechnique.

Mots-clés : sécheresse, été 2003, retralt gonflement des sols argileux, dommages au bâti. commission Cat-Nat, réglementations.

\section{Lessons drawn from the 2003 drought}




\section{Introduction et particularité de l'été 2003}

I'été 2003 a été caractérisé par des températures estivales exceptionnelles : des températures dépassant $35^{\circ} \mathrm{C}$ ont été mesurées dans deux tiers des stations météorologiques dans 180 communes. Des températures dépassant $40^{\circ} \mathrm{C}$ ont été mesurées dans $15 \%$ des stations, y compris en Bretagne, ce qui n'était jamais arrivé depuis le débul des mesures météorologiques.

Les désordres sur les constructions sont apparus dès le moís de juillet 2003. Or si l'on considérait les critères utilisés par la Commission de reconnaissance de l'état de catastrophe naturelle, l'année 2003 , ne présentait que pour très peu de départements les caractéristiques météorologiques d'une sécheresse. En effet, le critère météo employé par la commission avait, pour une de ses conditions, une a sécheresse hivernale w, c'est-à-dire sur une faible pluviosité en période de recharge hydrique des sols et des nappes d'eau souterraine. Or, l'hiver 2002-2003 fut plutôt pluvieux, et la plupart des départements présentaient une bonne recharge hydrique en sortie d'hiver.

Dès l'été 2003, une réflexion interministérielle a été lancée afin d'appréhender le phénomène observé, à savoir l'apparition de nombreux désordres aux constructions.

Il faut rappeler la nature du phénomène à l'origine des désordres au bâti et les élểments à prendre en compte pour caractériser ce que l'on appelle la u sécheresse géotechnique ». Ce phénomène ne concerne que les terrains caractérisés par des sols rỉches en certains minéraux argileux qui ont des propriétés de variation de volume en fonction de la teneur en eau. Ces variations de volume se traduisent à l"échelle macroscopique par des phénomènes de retrait en période sèche et de gonflement en période humidé. En climat tempếré, les argiles sont souvent proches de leur étal de saturation, ce qui explique que les mouvements observés sont plus importants en période sèche. La tranche superficielle du sol ( $1 \mathrm{~m}$ à $2 \mathrm{~m}$ de profondeur, volre davantage) est soumise à l'évaporation en période sèche, i] en résulte un retrait des argiles cư se manifeste par un tassement vertical et l'apparition de fissures verticales. L'amplitude de ce retrait est d'autant phus importante que la couche de matériau argileux est épaisse et qu'elle est riche er minéraux gonflants.

Les désordres liés à ces mouvements de terrain sont pris en compte par le régime de Catastrophe Naturelle depuis 1989 (Figure 1). L'étude des reconnaissances de l'état de catastrophe naturelle pour ce phénomène. nous permet d'établir un état des lieux des zones les plus touchées en France.

Les données communiquées par la Caisse centrale de réassurance (CCR) en octobre 2003, indiquent que la région parisienne est tout particulièrement concernée par le phénomène, puisque les sept départements de la région Ile-de-France (hors Paris) font partie des dix-sept départements pour lesquels les couts cumulés d'indemnisation pour des sinistres liés au retrait/gonflement des argiles sont les plus élevés. Viennent ensuite la Haute-Garonne, les Bouches-du-Rhône, le Nord, le Puy-de-Dôme, le Loiret et le Gers.

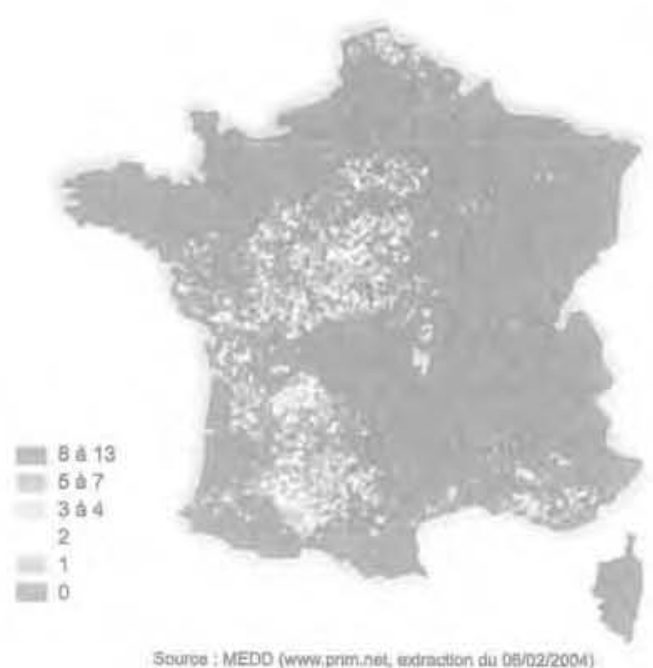

FG. N Nombre d'arTêtẻs Cat-Nat sécheresse par commune 1989-2004 (arrêtés relatifs à la sécheresse 2003 non pris en compte) Numbers of Cat-Nat orders per district $1969-2004$ (orders related to the 2003 drought being not taken tnto account)

\section{Présentation de l'ampleur du phéno- mène avant la sécheresse de 2003}

Les principales sécheresses prises en compte en France par une reconnaissance de l'état de catastrophe naturelle se sont déroulées au cours des années 1989-1992, et 1996-1997. Cés sécheresses ont touché 5000 communes réparties sur 75 départements. Le coût des indemnisations des désordres liés ì ces évènements s'élève a 3,3 milliards d'euros, ce qui place ce risque en deuxième position cerrière les inondations pour les indemnisations. Le coût moyen d'un sinistre est de 100006

Les désordres observés sont l'apparition de fissures en façade, souvent obliques, et passant par les points de faiblesse que constituent les ouvertures. Les désordres se manifestent également par des décollements entre éléments jointifs (garages, perrons, terrasses), ainsi que par une distorsion des portes et des fenếtres, une distocation des dallages, et des cloisons et, parfois la rupture de canalisations enter'ées (ce qui vient aggraver les désordres car les fuites d'eau qui en resultent provoquent localement des gonflements des sols). Les bâtiments qui sont les plus touchés par ce phénomène sont principalement des maisons individuelles fondées superficiellement, avec une structure en maçonnerie sans chaînage horizontal. Un facteur aggravant supplémentaire, est la présence d'un soussol partiel, ainsi que de la végétation à proximité de la construction. La présence de végétation à proximité des fondations joue un rôle très important sur l'ampleur des désordres observés, car elle contribue as assécher en profondeur les terrains argileux et augmente par conséquent l'ampleur du phénomène de variation de volume des terrains.

La manifestation de ce mouvement de sol engendrant des désordres sur les constructions est lié à deux types de facteurs : d'une part des facteurs déclenchants 
et d'autre part des facteurs aggravants. Les facteurs déclenchants sort liés directement à $\mathrm{la} \mathrm{météo,} \mathrm{ainsi}$ qu'à la nature des sols. En matière de prévention, mís à part la caractérisation de ces paramètres pour évaluer les zones les plus sensibles, il est difficile de les réduire pour limiter l'aléa. Par contre, un ensemble de facteurs aggravants sont maintenant connus et souvent en cause dans l'apparition des désordres, alors qu'il existe des solutions pour s'en affranchir. Ces facteurs sont constitués par des éléments naturels en rapport avec l'environnement de la construction: végétation, présence d'eaux souterraines, pente, ainsi que par des éléments anthropiques en rapport avec des laiblesses structurelles de la construction ou une iradaptation des fondations au terrain.

Les spécialistes du domaine s'accordent pour dire qu'il est techniquement possible de construire en France des bâtiments résistant au phénomène dé " sécheresse géotechnique on prenant des mesures adaptées concernant à la fois l'environnement immé. diat de la construction, et la structure même des bâtiments, et ce pour un surcoût faible à la construction. De plus, il faut considérer que les techniques recommandées pour s'affranchir du risque de dommages liês à ce phénomène assurent une meilleure a construction : construction plus durable, prescriptions concernant le gros œuvre qui vont dans le même sens que celles concernanl le bâti parasismique.

Compte tenu de cette possibilité technique de s'affranchir dans la plupart des cas des désordres, et du coût engendré par ce phénomène lors des dernières périodes de sécheresse connues en France, le ministère de l'écologie tente de mettre en place un dispositif de prévention pour limiter ce risque naturel.

\section{3}

\section{Actions de prévention engagées par le ministère de l'Ecologie}

Trois actions principales de prévention sont engagées, portant sur l'amélioration de la connaissance de l"alëa, l'information et la mise en place de plans de prévention des risques liés au phénomène de retrait gonflement des argiles.

\section{1}

\section{La connaissance de l'aléa}

Cette action, menée par le MEDAD, se décline ellemêrme en plusieurs sous-actions

- une sous-action visant à améliorer la connaissance de la manifestation du phénomène pour adapter au mieux les technicues pour réduire le risque et mieux caractériser les phénomènes dếclenchants (en particulier les conditions météorologiques permettant de distinguer les sécheresses anormales permettant d'améliorer le critère employé par la Commission interministérielle catastrophes naturelles):

- une sous-action de cartographie nationale de l'aléa retrait/gonflement des argiles, en cours de réalisation, ayant pour objectif de déterminer les zones à priori sensibles et d'y diffuser des règles de prévention. Cette politique de connaissance de la répartition géographique sur l'ensemble du territoire f̂rançajs de l'aléa u sécheresse géotechnique a est motivée par la connaissance technique qui existe pour s'affranchir pour les constructions nouvelles des désordres liés à ce risque, en respectant certaines règles de construction et certaines prescriptions concernant l'ervironnement immédiat de la construction. Cette cartographie de l'aléa consiste en la délimitation des principales zones concernées afin que, sur ces zones, soient mises en œuvre des mesures de prévention. Le BRGM est chargế de concrétiser ce programme de cartographie nationale de l'aléa a retrait/gonflement $n$ des argiles selon une méthodologie validée par le MEDAD : cartographie à l'échelle départementale, au 1/50 000, se basant sur la carte géologicue et la caractérisation des couches superficielles vis-à-vis du phénomène de retrait/gonflement. II s'agit en effet de recenser et de hiérarchiser les zones les plus sensibles. A l'heure actuelle 34 cartes d'aléas ont été finalisées (au 19/09/2007) et sont consultables et téléchargeables sur le site: www.argiles,fr. La cartographie de l'ensemble du territoire métropolitain est prévue pour fín 2010. Les départements les plus sinistrés en terme de coût des désordres et de nombre de reconnaissance de l'état de catastrophe naturelle lié à ce risque onl été cartographiés en premier.

\section{2}

\section{L'information}

Il existe plusieurs axes d'information concernant les risques naturels et plus particulièrement les risques liés à la sécheresse géotechnique.

La filiěre administrative doil d’abord être citée. L'information des citoyens sur les risques naturels et technologiques majeurs auxquels ils sont soumis dans certaines zones du territoire et sur les mesures de sauvegarde qui les concernent est un droit inscrit dans le code de l'environnement aux articles L 125-2, L 125-5 et L 563-3 et R $125-9$ à R 125-27. L'information préventive dans le cadre de celte loi concerne trois niveaux de responsabilité : le préfet, le maire et le citoyen en tant que gestionnaire, vendeur ou bailleur.

En plus de cette information prevue dans le cadre de la loi, le MEDAD met en place d'autres moyens d'information des particuliers pour le risque de sécheresse géotechnique, notamment les cartes d'aléa réalisées par le BRGM présentées cì-dessus.

Prochainement des fiches $\alpha$ mitigations a seront téléchargeables sur le site www.prim.net. Ces fiches décrivent les différentes mesures et techniques concernant à la fois le bâtì et l'environnement immédiat de la construction qu'il est conseillé de mettre en aeuvre lorsque notre habilat ou notre projet se situe sur un terrain sensible au retrait/gonflement des argiles.

En collaboration avec le ministère de l'Équipement et l'Agence Qualité Construction, le MEDAD a réalisé un outil de communication à destination des professionnels de la construction des maisons individuelles pour diffuser les bonnes pratiques à respecter lorsque j’on construit sur un terrain sensible. Plusieurs réunions régionales rassemblant plusieurs centaines de professionnels se sont tenues pendant l'année 2005 .

Beaucoup de services départementaux de l'Élat diffusent, après validation de la carte d"aléa du BRGM, des plaquettes d'information à destination des particuliers souhaitant construire pour les sensibiliser à l'existence de ce risque. 


\section{La mise en place des PPR retrait/gonflement des argiles}

En l'absence d'une réglementation nationale concernant des règles de construction sur les terrains sensibles au retrait/gonflement des argiles, le MEDAD a mis en place des PPR ayant pour objectif de diminuer les désordres liés à ce phénomène. Une question préalable à cette démarche a été de savoir si ce risque constituait un risque majeur. En effet ce risque est certes très coûteux en teme de réparation de désordres, mais n'a pour l'instant pas provoqué de dommages humains.

La démarche de mise en ceuvre de PPR a séche resse $i$ a deux objectifs : d'une part ce dispositif permet de diffuser des connaissances et d'éveiller une a conscience du risque n, notamment grâce à l'information acquéreur-locataire désormais obligatoire. D'autre part, le PPR rend obligatoire des prescriptions permettant de diminuer le risque sur les zones sensibles. La politique de prescription de PPR sécheresse vise aujourd'huj à traiter par un mểme PPR l'enseñble des zones concernées par l'aléa au sein du même département. Cette démarche a pour objectif de mettre en geuve rapidement une prévention de ce risque dans les zones où l'on dispose d'une information sur la répartition géographique de l'aléa, et d'éviter de contjnuer de " $\mathrm{mal}$ y construire sur ces zones.

Afin de faciliter la mise en ceuvre des PPR c séche. resse $),$ et d'optimiser les mesures de prêvention, la DPPR a réuni un groupe de travail regroupant à la fois des services en charge de la réalisation des PPR, des géotechniciens, des assureurs, des professionnels de la construction et d'autres experts. Ce groupe de travall a pour but d'améliorer le modèle de règlement type, fort de l'avancée des connaissances techniques et scientifiques et du retour d'expérience des services de l'État sur les PPR déjà approuvés. Les interrogations portent actuellement sur l'articulation des différents a axes de prévention visant à agir sur la construction et sur l'environnement immédiat de celle-ci

En parallèle de ces actions de prévention, le MEDAD intervient également dans la procédure de régime d'indemnisation de la réparation des désordres liés à ce risque en tant qu'expert technique au sein de la Commission interministérielle de déclaration de l'état de catastrophe naturelle.

\section{4}

\section{Zoom sur la situation particulière de la sécheresse de l'été 2003}

Dès juillet 2003, le constat de l'apparition de nombreux désordres aux constructions est réalisé. Au cours de l'été apparaissent des désordres très importants qui ont, pour certains d'entre eux, conduit des maires à prendre des arrêtés de périls. Mais, compte tenu de l'hiver 2002-2003 assez pluvieux, malgré cette observation des désordres, ce phénomène ne correspondait pas au critère météo utilisé par la Commission de l'état de catastruphe naturelle pour caractériser un phénomène de sécheresse exceptionnelle.
Le régime d'indemnisation des désordres relève des catastrophes naturelles, garantie incluse dans les polices multirisques habitation prises par les maîtres d’ouvrage. La loi n $82-600$ du 13 julllet 1982 a fixé pour objectif d'indemniser les victimes de catastrophes naturelles en se fondant sur le principe de solidarité nationale. Cette loi concerne les phénomènes : inondations, mouvements de terrains et du sol, séismes, avalanches, submersion marines el cyclones très intenses. Afin de pouvoir signer ou non un arrêté de déclaration Cat-Nat (finances, intérieur), une commission consultative statue sur « l'intensité anormale de l'agent naturel \%. La loi du 31/07/2003 a aussi confirme la franchise obligatoire dont le montant, fixé par arrêté, est de $1520 €$ en cas de dommages dus à la sécheresse ou lạ réhydratation des sols, alors qu'elle est de $380 €$ pour les autres risques naturels. En absence de PPR prescrit ou approuvé sur une commune, si celle-ci fait l'objet de plusieurs reconnaissances pour un même type de phénomène, cette franchise double à partir du deuxième arrêté, trịple ensuite

\section{5}

\section{Présentation succincte du fonction- nement du système " Cat-Nat "}

Après la survenance d'un phénomène naturel non assurable et pris en compte dans le régime Cat-Nat, les particuliers transmettent un dossier au maire de leur commune. Les maires le font suivre ensutte au préfet, accompagné d'une demande de reconnaissance en état de catastrophe naturelle s'ils jugent que le phénomène observé sur leur commune releve de cette instruction. Les dossiers sont instruits par la préfecture : sollicitation des expertises nécessaires, vérification du contenu des dossiers - recensement des études nécessaires et transmission des dossiers complets à la DDSC (direction de la Défense et de la Sécurité civile) du ministère de l’Intérieur. Le ministère de l'Intérieur préside cette commission ef assure la transmission des dossiers aux différents membres de la commission : direction du Budget et dírection du Trésor pour le ministère des Finances, le ministère de l'Outre-Mer quand des demandes proviennent des DOM-TOM, el le ministère de l'Écologie (qui ne vote pas, mais qui a un rôle d'expert technicque sur les dossiers). Une réunion interministérielle de déclaration de l'état de catastrophe naturelle se réunit une fois par mois. Cette commission émet un avis sur les dossiers. A partir de cet avis, les ministres de l'intérieur et des Finances prennent une décision qui paraît au J.O. Une fois l'arrêté paru au J.0. les citoyens ont un délai de 10 jours pour déclarer un sinistre auprès de leur assurance. Le rôle des experts d'assurance est ensuite de vérifier le lín entre l'agent naturel (le plénomène observé) et la survenance des dommages.

Atin d'être éligible pour le phénomène de retraitgonflement des argiles, deux questions se posent :

- d'une part, est-ce çue l'intensité de la sécheresse peut. être qualifiée d'anormale?

- d’autre part, existe-t-il un rapport de causalité entre l'apparition des dommages et la secheresse? Cette question amène trois sous-questions : y a-t-il corrélation temporelle entre la sécheresse observée et l'apparition de dommages? Les sols de fondation des constructions endommagées sont-ils sensibles au phé- 
nomène de dessiccation et ont-îls été affectés par celuíci ? Peut-on éliminer toute cause non naturelle susceptible de provoquer des effets similaires sur le bâti ?

Pour répondre à la première de ces questions, la Commission s'est dotée, en 2000, d'un critère météorologique. Ce sont les experts des assurances qui auront ensuite à répondre à la deuxième question, la commission demande uniquement, en ce qui concerne la nature du sol une étude de sol attestant de la présence d'argile sur la commune, pour les communes dont c'est la première demande de reconnaissance de l'état de catastrophe naturelle pour ce type de phénomène. L'éligibilité d'une commune est donc fondée essentiellement sur l'ntensité météorologique de la sécheresse observée.

\section{6}

\section{Particularité de la sécheresse 2003}

La sécheresse qui a été observée pendant l"été 2003 est liée à un déficit hydrique survenu après l'hiver. Le précédent critère météo de la commission Cat-Nat, évoqué ci-dessus, avalt powr une de ces conditions un déficit hydrique en période de recharge hivernale, ce qui n'a pas été le cas de l'hiver 2003. Par conséquent, ce critère ne traduisait pas la sécheresse subie par les constructions en 2003. Une réflexion a été menée par les différents services afin de modifier ce critère pour prendre en compte la sécheresse exceptionnelle de 2003. La recherche d'un nouveau critère expliquue en partie le délai de traitement des demandes de reconnaissance de l'état de catastrophe naturelle pour les dommages causés par les mouvements de terrain différentiels consécutifs à la sécheresse et à la réhydratation des sols survenus pendant l'été 2003.

La sécheresse 2003, s'est essentiellement distinguée des sécheresses antérieures par son caractère très concentré dans le temps (on a parlé du a coup de chaleur n de l'été 2003) et, surtout, par le fait qu'elle n'avait pas été précédée d'une pluviosité anormalement basse au cours des mois de l'hiver précédent (janvier-mars 2003), alors que le critère appliqué depuis les années 1999-2000 pour caractériser sur le plan météorologique les sêcheresses du sol d'une intensité anormale était justement fondé sur ce phénomène de « choc hivernal w.

Dès la fin de la canicule 2003, les services de letat ont essayé de caractériser au mieux et le plus justement possible le phénomène observé. En effet, compte tenu de l'cnsemble des désordres observés, il était urgent de caractériser ce phénomène. La démarche mise en ceuvre pour cela a été d'élaborer un nouveau critère météorologique quj permette de qualifier l'intensité anormale de la sécheresse : afin de savoir sil les critères proposés étaient représentatìs du phénomène observé, des comparaisons ont été réalisées avec les critères envisagés et les cartes des dommages recensés. Parmi les inconnues techniques pour déterminer ce nouveau critère, on peut citer par exemple les questions sur l'ori. gine des désordres observés : sont-ils dus à la forte chaleur, ou bien à des effets cumulatífs liés aux sécheresses précédentes, ou encore à la manifestation d'effets différés, les désordres liés au retrait des argiles survenant souvent après la fin de la période de sécheresse.

En pratique, ces difficultés pour définir un nouveau critère se sont traduites par des reconnaissances et donc des indemnisations tardives (les deux premiers arrêtés sont datés des 25 août 2004 et 11 janvier 2005), ainsi que de quelques révisions puisque le critère météorologique adopté au départ a été assoupli par la suite et donc par conséquent, il a fallu reprendre les dossiers rejetés auparavant afin de vérifier s'ils répondaient au nouveau critère.

Pour déterminer ce nouveau critère, plusieurs réunions interministérielles se sont tenues ả partir du mols d'août 2003.

Par ailleurs, suite aux difficultés rencontrées dans le traitement de ce phénomène et aux questions géné rales que posait le régime Cat-Nat, une mission d'inspection a été lancée en février 2005 par les ministres de I'Intérieur, des Finances et de l'Écologie et du Développement durable pour établir un état des lieux du régine des victimes des catastrophes naturelles et faire des propositions en vue de sa réforme éventuelle. Dans ce cadre, un examen particulier du dossier de la sécheresse de l'été 2003 avait été demandé prioritairement et dans des délais rapides.

Enfin, pour les personnes fortement sinistrées, mats dont la commune ne vérifiait pas les nouveaux critères météorologiques arrêtés par la commission Cat-Nat pour traiter du phénomène de l'été 2003, une procédure exceptionnelle a êté mise en place. Cette procédure concerne les dommages aux bâtiments causés par la sécheresse survenue entre juillet et septembre 2003 et la réhydratation des sols quj lui a été consécutive, lorsque ceux-ci compromettent la solidité des bâtiments ou les rendent impropres à leur destination. Cette procédure est réservée aux propriétaires des bâtiments à usage d'habitation principale, situés dans les communes qui ont formule, avant le $1^{\text {er }}$ juin 2005 , une demande de reconnaissance de l'état de catastrophe naturelle prévue aux articles L. 125-1 et suivants du code des assurances au tire de la sécheresse survenue entre juillet et septembre 2003 et qui ne l'on' pas obtenue. Les aídes portent exclusivement sur les mesures de confortement nécessaires au rétablissement de l'íntégrité de la structure, du clos et du couvert.

\section{7}

\section{Conclusion sur la sécheresse 2003 en chiffres : premières estimations}

La sécheresse géotechnique (aléa retrait/gonflement des argiles) ayant frappé la France, l'été 2003. a provoqué des désordres coûteux aux constructions entraînant 8172 demandes de communes pour un classement en état de catastrophe naturelle (seuls cing départements n'ont pas demandé d'arrêtés de catastrophes naturelles) auxquels il a été donné une réponse favorable dans 4274 cas en date de juin 2007. De plus, le gouvernement a mis en place une procédure exceptionnelle d'indemnisation hors procédure Cat-Nat. Le coût de cette sécheresse pour le marché de l'assurance est de l'ordre de 1,5 milliard d'euros. Si nous additionnons le coût de cette sécheresse à celles des années 90, le coût des dommages moyens annuels dépasse 300 mililions d'euros. 